\title{
A Novel Protocol for Streamlined IRB Review of Practice-based Research Network (PBRN) Card Studies
}

\author{
Michelle D. Hamilton, BA, Philip A. Cola, MA, Joshua J. Terchek, BA, \\ James J. Werner, PhD, and Kurt C. Stange, MD, PhD
}

Purpose: The "card study," in which clinicians record brief information about patient visits during usual clinical care, has long been a rapid method for conducting descriptive studies in practice-based research networks. Because an increasingly stringent regulatory environment has made conducting card studies difficult, we developed a streamlined method for obtaining card study institutional review board (IRB) approval.

Methods: We developed a protocol for a study of the card study method, allowing new card study proposals of specific research questions to be submitted as addenda to the approved Card Study Protocol.

Results: Seven card studies were proposed and approved under the Card Study Protocol during the first year after implementation, contrasted with one-card study proposed in the previous year. New card study ideas submitted as addenda to an approved protocol appeared to increase IRB comfort with the card study as a minimal risk method while reducing the hurdles to developing new study ideas.

Conclusions: A Card Study Protocol allowing new study questions to be submitted as addenda decreases time between idea generation and IRB approval. Shortened turn-around times may be useful for translating ideas into action while reducing regulatory burden. (J Am Board Fam Med 2011;24:605-609.)

Keywords: Card Study, Institutional Review Board, Practice-based Research Network, Practice-based Research, Primary Health Care.

The majority of research conducted in the United States is basic science investigation conducted in academic medical centers. ${ }^{1}$ Discoveries from this research can take years or decades to translate into clinical practice $^{2}$ and are often not directly applicable to clinical practice because research conducted in highly controlled settings differs greatly from community medical offices, the setting where most medical care is delivered to most patients. ${ }^{3,4}$

This article was externally peer reviewed.

Submitted 1 February 2011; revised 15 June 2011; accepted 22 June 2011.

From Case Western Reserve University (JJW), Department of Family Medicine, Research Division (MDH, JJT), and Epidemiology and Biostatistics, Sociology, and Oncology (KCS); and Research and Technology, University Hospitals Case Medical Center, Case Western Reserve University (PAC), Cleveland, Ohio.

Funding: none.

Conflict of interest: none.

Corresponding author: Michelle Hamilton, BA, Case Western Reserve University, Department of Family Medicine, Research Division, 10900 Euclid Avenue, Cleveland, OH 44106 (E-mail: michelle.hamilton@case.edu).
In response to concerns about the relevance and slow translation into practice of most research, two threads of solutions have emerged. One thread is the practice-based research network (PBRN), in which clinicians on the front lines of health care band together to generate relevant new knowledge. ${ }^{5-12}$ Another more recent development is the National Institutes of Health Clinical and Translational Science Award (CTSA),${ }^{13-15}$ which aims to transform the way research is conducted and translated into practice and community applications. ${ }^{10,13-15}$ Many CTSAs involve collaboration with PBRNs. ${ }^{16}$

\section{The Card Study and the Research Regulatory Environment}

A staple PBRN study method developed by the Ambulatory Sentinel Practice Network (ASPN) more than two decades ago is the weekly return card, ${ }^{17,18}$ or "card study." ${ }^{19}$ In card studies, a question about clinical practice is refined by the network into a short series of observational data ele- 
ments that can be quickly recorded on a pocketsized data collection card during the course of routine practice. ${ }^{19}$ Card studies are used by many PBRNs for rapid turn-around pilot studies and have made important contributions of new knowledge to the published literature. ${ }^{19-24}$ Example study cards are included in the On-line Appendix and in Figure 1.

Institutional review boards (IRBs) must review research protocols to ensure that the research is ethical and protects the rights and welfare of human subject participants. ${ }^{25}$ However, IRB review can be a difficult process for practice-based researchers, as these researchers struggle to learn and apply unfamiliar rules regarding research. ${ }^{16} \mathrm{~A}$ rapid cycle between raising and answering PBRN questions facilitates ongoing clinician engagement. The wide variation in how IRBs review research, ${ }^{26}$ including observational research studies, ${ }^{27}$ creates confusion and can create a bottleneck in the PBRN research process. ${ }^{28,29}$

The card study has traditionally been a low-risk method that often received expedited IRB review. However, the research regulatory environment has become more stringent ${ }^{30}$ since the card study method was first developed, increasing barriers to conducting PBRN card studies. Additional privacy regulations imposed by the Health Insurance Por-

Figure 1. Identification of developmental delays card study data collection card.

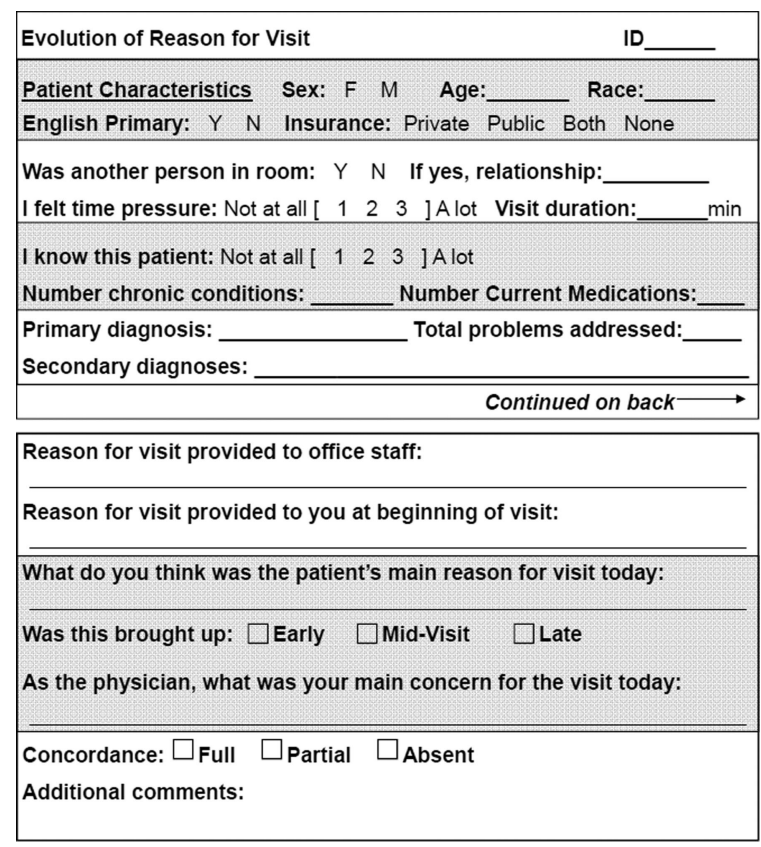

tability and Accountability Act (HIPAA) ${ }^{31}$ along with increased regulatory scrutiny cause IRBs to raise their threshold of concern, even for minimal risk observational studies, such as card studies. ${ }^{32-37}$ This challenge causes researchers and clinicians to modify study ideas, or forgo certain study designs altogether when IRB submission is viewed as "not worth the effort." ${ }^{37}$

Streamlining the process of clinical and translational research while protecting participants is a major focus of CTSAs, and many have dedicated shared resources to research regulation. In Cleveland, the Clinical and Translational Science Collaborative worked to engage the experience of both our PBRN and Regulatory Shared Resources to develop, implement, and evaluate a novel approach to streamline the process of IRB approval for card studies. This paper describes that process and includes an appendix that can be adapted by other PBRNs, CTSAs, and IRBs seeking to meet the joint aims of protecting research participants, developing PBRNs, and speeding the translation of clinical research questions into investigation and dissemination.

\section{Methods}

\section{Development of a Single, Streamlined IRB Protocol for Card Studies}

Given this clinical and regulatory environment, the PBRN Shared Resource began to investigate facilitation of efficient turnaround of card studies. In brainstorming with the CTSA Head of Regulatory Knowledge, a study of the card study method was suggested as a possible method for allowing easy review of specific card studies asking new study questions.

\section{The Card Study Protocol}

A parent study of the card study method, the Card Study Protocol, was developed by the PBRN Shared Resource. The objective of the Card Study Protocol is to understand the uptake of different card studies. The Card Study Protocol outlines a set of requirements for low-risk card studies, such as collection of de-identified data, and describes a standard set of procedures including recruitment methods, data collection, data management, and privacy procedures. Specific new card studies are then added as addenda to the parent protocol, so 
long as the individual card studies fit the standard criteria.

The Card Study Protocol modifies the traditional process of conducting card studies through generating and submitting a protocol and IRB submission that must undergo new protocol review for each individual card study. The Card Study Protocol combines multiple de-identified and/or limited dataset (minimal risk) card studies into one continuous protocol, under one principal investigator. As new card studies are proposed, the Card Study Protocol is amended to include additional research questions and the clinician or researcher who proposes the new card study idea is added as a coinvestigator to the parent protocol.

\section{Results}

Combing all minimal risk card studies into one study of the card study method facilitated IRB review, resulting in increased efficiency as the IRB does not have to revisit the issue of what a card study is each time an additional question is submitted. In addition, review of amendments is a more efficient process focused almost exclusively on "changes" compared with new protocol reviews requiring review of all aspects of a proposed study, including methods previously approved under separate IRB applications. The complete Card Study Protocol is included in the On-line Appendix for those who wish to use this as a model for their own IRB applications.

The Card Study Protocol was submitted for IRB review in March of 2009 and approved in June of 2009, a quicker process than usual, attributed to meetings with the IRB regarding the proposed Card Study Protocol before submission. Seven card studies were proposed and approved as part of the protocol during the first year of use, compared with the previous year, in which one card study was proposed but not submitted to the IRB or implemented. The most recent card study addendum received approval in seven calendar days, compared with similar risk studies not tied to the Card Study Protocol having previously taken nearly 1 year to receive approval.

In addition, we are finding that the threshold for proposing ideas is reduced for PBRN members as well as academic health center investigators because of a sense that new ideas have a chance to get into the field quickly, while enthusiasm from initial idea generation is fresh. Similarly, IRB staff and members report that seeing new study ideas as addenda to a protocol that has already been reviewed and judged to be minimal risk makes it easier to understand the context and minimal risk nature of new card studies. This familiarity with the method increases the likelihood of identifying and determining the particular risks and benefits of the new question, data, and study sample.

Although card studies under the Card Study Protocol are limited to low risk studies involving de-identified data, a wide range of study types are still possible. Card studies approved under the Card Study Protocol have included exploring patient visits, such as developmental surveillance in well-child health visits, the frequency of patients presenting information gathered on-line before visits, patients mentioning direct to consumer advertising in visits, and patients raising dental concerns during medical visits. A survey study of clinicians has also been included, stretching the notion of cards to include large, two-sided data collection forms.

\section{Discussion}

Engaging clinicians, patients, and questions from real-world practice are vital to efforts to increase the relevance and utility of clinical research. Card studies are a tried and true method for accomplishing this, with continued relevance to complement or be applied to electronic data collection. ${ }^{19,38} \mathrm{We}$ have found the Card Study Protocol described here to be feasible and useful in educating IRBs and PBRNs about each other, while facilitating safe and timely clinical research.

This Card Study Protocol is not appropriate for greater than minimal risk studies that may use extensions of the card study method. ${ }^{19}$ Such studies should undergo individual review. Our evaluation of this protocol is informal and based on a single IRB and handful of local PBRNs over a short period. However, we believe that this early experience and the face value of the idea of studying the card study method through a single protocol in which additional minimal risk study questions are introduced through addenda justify sharing this idea with the PBRN and research regulatory communities so that additional experience can be gained and immediate benefits we observed can be realized by others. Further research should examine the longer-term effects of the Card Study Protocol on the 
relationships between PBRN investigators, IRB staff, and relevant IRB members, as well as on protocol review time, participant safety, and the generation of new knowledge relevant to clinical practice. This work embodies a goal of the CTSA, to reduce regulatory burden for investigators to promote the conduct and growth of clinical/translational research. ${ }^{13-15,39}$

This publication was made possible by the Case Western Reserve University/Cleveland Clinic CTSA Grant No. UL1 RR024989 from the National Center for Research Resources (NCRR), a component of the National Institutes of Health and NIH roadmap for Medical Research and the Practice-based Research Network Core facility of the Case Comprehensive Cancer Center (P30 CA43703). Its contents are solely the responsibility of the authors and do not necessarily represent the official view of NCRR or NIH. Dr. Stange's time was supported in part by a Clinical Research Professorship from the American Cancer Society.

\section{References}

1. Green LA, Fryer GE Jr, Yawn BP, et al. The ecology of medical care revisited. N Engl J Med 2001;344: 2021-25.

2. Balas EA, Boren SA. Managing clinical knowledge for health care improvement. In: Bemmel J, McCray AT, editors. Yearbook of Medical Informatics 2000: Patient-Centered Systems. Stuttgart, Germany: Schattauer Verlagsgesellschaft mbH; 2000;65-70.

3. Nutting PA, Green LA. Practice-based research networks: reuniting practice and research around the problems most of the people have most of the time. J Fam Pract 1994;38:335-6.

4. Fortin M, Dionne J, Pinho G, et al. Randomized controlled trials: do they have external validity for patients with multiple comorbidities? Ann Fam Med 2006;4:104-8.

5. Nutting PA. Practice-based research: laboratories for outcomes and effectiveness research. Paper presented at: AHCPR Conference Proceedings. Primary Care Research: Theory and Methods,1991; Washington, DC.

6. Nutting PA, Green LA. And the evidence continues to establish the feasibility of practice-based research. Fam Med 1993;25:434-6.

7. Nutting PA, Beasley JW, Werner JJ. Practice-based research networks answer primary care questions. JAMA 1999;281:686-8.

8. AHRQ Practice-based Research Networks (PBRNs). http://www.ahrq.gov/research/pbrn/pbrnfact.htm. Accessed June 30, 2010.

9. Fagnan LJ, Handley MA, Rollins N, Mold J. Voices from left of the dial: reflections of practice-based researchers. J Am Board Fam Med 2010;23:442-51.
10. Westfall JM, Mold J, Fagnan L. Practice-based research: "Blue Highways" on the NIH roadmap. JAMA 2007; 297:403-6.

11. Green LA, Hames CG Sr, Nutting PA. Potential of practice-based research networks: experiences from ASPN. Ambulatory Sentinel Practice Network. J Fam Pract 1994;38:400-6.

12. Green LA, Dovey SM. Practice based primary care research networks: they work and are ready for full development and support. BMJ 2001;322:567-8.

13. Reis, SE, Berglund, L, Bernard, GR, et al. Reengineering the national clinical and translational research enterprise: the strategic plan of the national clinical and translational science awards consortium. Acad Med 2010;85:463-9.

14. Califf, RM, Berglund, L. Linking scientific discovery and better health for the nation: the first three years of the NIH's Clinical and Translational Science Awards. Acad Med 2010;85:457-62.

15. Waldman, SA, Terzic, A. Clinical and translational science: from bench-bedside to global village. Clin Translational Sci 2010;3:254-7.

16. Fagan LJ, Davis M, Deyo RA, et al. Linking practice-based research networks and Clinical and Transitional Science Awards: new opportunities for community engagement by academic health centers. Acad Med 2010;85:476-83.

17. Green LA. The weekly return as a practical instrument for data collection in office based research. Fam Med 1988;20:182-4.

18. Green LA, Reed FM, Miller RS, Iverson DC. Verification of data reported by practices for a study of spontaneous abortion. Fam Med 1988;20:189-91.

19. Westfall JM, Zittleman LK, Staton EW, et al. Card studies for observational research in practice. Ann Fam Med 2011;9:63-88.

20. Flocke SA, Orzano AJ, Selinger HA, et al. Does managed care restrictiveness affect the perceived quality of primary care? A report from ASPN: Ambulatory Sentinel Practice Network. J Fam Pract 1999;48:762-8.

21. Vinson DC, Manning BK, Galliher JM, et al. Alcohol and sleep problems in primary care patients: a report from the AAFP National Research Network. Ann Fam Med 1010;8:484-92.

22. Vinson DC, Galliher JM, Reidinger C, Kappus JA. Comfortably engaging: which approach to alcohol screening should we use? Ann Fam Med 2004;2: $398-404$.

23. Froom J, Culpepper L, Grob P, et al. Diagnosis and antibiotic treatment of acute otitis media: report from International Primary Care Network. BMJ 1990;300:582-6.

24. Froom J, Culpepper L, Green LA, et al. A crossnational study of acute otitis media: risk factors, severity, and treatment at initial visit: report from the International Primary Care Network (IPCN) 
and the Ambulatory Sentinel Practice Network (ASPN). J Am Board Fam Pract 2001;14:406-17.

25. Protection of Human Subjects: Title 45, Code of Federal Regulations, Part 46. Office for Human Research Protections (OHRP), HHS.

26. Grady C. Do IRBs protect human research participants? JAMA 2010;304:1122-3.

27. Green LA, Lowery JC, Kowalski CP, Wyszewianski L. Impact of institutional review board practice variation on observational health services research. Health Serv Res 2006;41:214-30.

28. Pace WD, Staton EW, Holcomb S. Practice-based research network studies in the age of HIPAA. Ann Fam Med 2005;3(Suppl 1):S38-45.

29. Wolf LE, Croughan M, Lo B. The challenges of IRB review and human subjects protections in practice-based research. Med Care 2002;40:521-9.

30. Baily MA. Harming through protection? N Engl J Med 2008;358:768-9.

31. HIPAA Privacy Rule: Information for Researchers. http://privacyruleandresearch.nih.gov/. Accessed January 1, 2011, 2004.
32. Nuremberg code, 1946. In Warren T. Reich, editor. Encyclopedia of Bioethics. Volume 4. New York: The Free Press; 1978:1764-5.

33. Shamoo AE. Future challenges to human subject protection. Scientist 2000;14:35-7.

34. Advisory Committee on Human Radiation Experiments (ACHRE). Final Report, Washington, DC: US Government Printing Office; 1995.

35. Office of Protection from Research Risks, "Letter of March 22, 1999, from OPRR to VA Greater Los Angeles Healthcare System.

36. Thompson, RE. Are human research subjects safe enough? Ethical aspects. Physician Exec 2003 (July).

37. Pentecost MJ. HIPAA and the law of unintended consequences. J Am Coll Radiol 2004;1:164-5.

38. Pace WD, Staton EW. Electronic data collection options for practice-based research networks. Ann Fam Med 2005;3(Suppl 1):S21-9.

39. Kim S, Ubel P, De Vries R. Pruning the regulatory tree. Nature 2009;457:534-5. 


\title{
A Novel Protocol for Streamlined IRB Review of Practice-based Research Network (PBRN) Card Studies
}

\author{
Michelle D. Hamilton, BA, Philip A. Cola, MA, Joshua J. Terchek, BA, \\ James J. Werner, PhD, and Kurt C. Stange, MD, PhD
}

\section{PBRN Shared Resource Practice-based Research Card Studies}

The following is the IRB protocol for the PBRN Shared Resource Card Study Protocol. This document is intended to be helpful to those who wish to implement similar protocols at other institutions.

Principal Investigator: James Werner, PhD; CoInvestigators: Catherine Demko, $\mathrm{PhD}$; Susan Flocke, PhD; Michelle Hamilton, CCRP; Peter Lawson, MA, MPH; Ash Seghal, MD; Kurt Stange, MD, PhD; Robin Strosaker, MD; Sharon Weyer, DNP, NP-C; and Steven Zyzanski, PhD.

\section{Introduction}

This protocol examines the card study research method, a study design widely used nationally and internationally to conduct simple, quick turnaround, low risk, and observational studies of usual medical practice in primary care practices that are part of practice-based research networks (PBRNs).

This is a participatory research project that provides clinicians and PBRN leaders with the opportunity to pose card study questions and to participate in refining questions and interpreting the findings at PBRN meetings.

Expedited review and waivers of both informed consent and HIPAA authorization are requested for this protocol.

\section{Background}

The majority of research conducted in the United States is basic science research conducted in academic medical centers. ${ }^{1}$ Discoveries in this branch of research can take years or even decades to reach clinical practice and are often not directly applicable because research conducted in a highly controlled setting differs greatly from the setting that primary care is delivered in. Clinicians often do not see the applicability of basic translational science research to their practice and may not be motivated to participate in this type of research. With the increased demands on clinicians, participation in research with little or no benefit may become increasingly difficult. A need for the ability to quickly translate basic research into improvements in clinical practice, patient experience and outcomes while engaging clinicians has been increasingly recognized. $^{2}$

\section{Practice-based Research Networks}

PBRNs are one setting where research relevant to practice is conducted. A PBRN is a group of ambulatory care practices whose primary goal is clinical care, but whom also participate in research projects to understand and improve clinical care. During the 1990s, PBRNs emerged as an important infrastructure for the study of health care and the translation of research into clinical practice in the United States. ${ }^{3}$ PBRNs can be an ideal setting to conduct primary care research that may quickly be translated into improvements in clinical care due to the collaboration with clinicians, access to large numbers of patients and feedback to clinicians that occurs in the research networks. The engagement of clinicians, and support staff is essential to the long-term success of research.

Creating added value for practicing clinicians and staff is among the most important challenges facing PBRNs. Part of the "value" lies in asking and answering questions important to member practices. PBRNs often further enhance value by allowing participating practices to learn about study findings quickly in easy-to-read feedback reports for clinicians and office staff that provide practicelevel and network-level data. In addition, practices are continuously polled, looking for ways of "giving 
back" to busy members. This enables PBRNs to foster a continuous reciprocal relationship between clinicians and investigators. $^{4}$

One goal of the PBRN Shared Resource is to provide member clinicians with a means to answer research questions relevant to their own clinical practice through the use of card studies.

\section{The Card Study}

The card study or "weekly return" card has been a staple of practice-based research since its inception in the Netherlands in the late 1960s and subsequent modification in the 1980s by the Ambulatory Sentinel Practice Network (ASPN) into its current 1-sheet (card), pocket-sized data collection form. ${ }^{5,6}$ Card studies describe what is occurring in clinical practice without changing regular clinical care.

Card studies are conducive to small, clinicianinitiated projects. In addition, PBRN-based card studies are an ideal method of conducting smallscale observational studies that involve clinicians, office staff, and patients, with the focus on minimizing the study's impact on the care processes. These studies sometimes are undertaken before embarking on a large-scale study as the flow of practice is an important "litmus test" of study feasibility. This approach increases the quality of data collection methods and results and also builds social capital among PBRN practices and staff. The concept of academic-based researchers relying on community-based providers for recruitment of participants, without further involving providers or patients in the design, conduct, or analysis of their studies is ultimately unsustainable. The clinical staff must be made to feel that their input is essential for enhancing the quality of the PBRN-based research. It is necessary for these busy practices to see the value in their research participation and to believe that it can enhance the quality of patient care and the care processes. ${ }^{4}$

It is essential that data collection methods fit the setting where the research is being conducted. For example, enrolling patients and obtaining their consent or implementing interventions often cannot be the responsibility of busy office staff or clinicians due to time constraints or other barriers. In contrast, clinicians are often receptive to being asked to collect specific data that can fit on a weekly return card as part of a card study. This method works best when data collection takes less than 2 minutes to complete per patient and a 1-page, simply written data collection protocol is included. ${ }^{4}$

Data collected via the card study method has been shown to be comparable in accuracy to data collected via other methods. ${ }^{7}$ However, this form of data collection differs from others in that it is simple and allows busy clinicians to participate in research studies that they may not have been able to take part in otherwise.

The card study method allows clinicians the ability to capture data concerning usual care without the intrusion of a research assistant in a way that is of minimal risk to patients as information may be entirely de-identified.

\section{Rationale and Significance of the Study Purpose}

Although the card study has a well-established place in practice-based primary care research, the current practice environment has changed considerably from the early days of the card study. Independent practices have largely been acquired by health systems, further increasing demands on clinician time and priorities. There are also new concerns for clinicians, such as increased patient privacy regulations. The changes in the practice environment leave less time than was available in the past for "extracurricular" endeavors, such as research. This study aims to characterize the implementation of card studies in the busy practices of today.

\section{Objectives}

This research project aims to study the uptake of card studies in the networks that fall under the PBRN Shared Resource to answer the following research questions:

1. Over a range of study questions, what percent of clinicians will volunteer to gather data?

2. Among enrolled clinicians, how many will successfully complete data collection?

3. How complete is the answering of specific card items over a range of study questions?

4. How many clinicians request feedback reports?

\section{Study Design}

Card study questions come from a variety of sources. Typically they come from questions that occur to PBRN clinicians during the course of 
seeing patients. Card study ideas can also come from PBRN directors or academic investigators. Questions and the protocol are typically refined by discussion with a group of PBRN members (often the network steering committee) and academic partners (often the PBRN Shared Resource). Often these questions result in the gathering of pilot data in preparation for larger studies.

\section{Population to be Studied}

Study participants will be practicing family clinicians who are part of the networks working with the Clinical and Translational Science Collaborative (CTSC) PBRN Shared Resource. These geographically-dispersed clinicians will record deidentified clinical observations regarding usual care of patients in their practice.

Please see Attachment C: Clinicians Who Will Be Invited to Participate in This Research Study (not included in this Appendix) for a list of all clinicians who may be invited to participate in this research (all PBRN members).

\section{Registration Procedures}

Potential participants will be identified through study interest sheets returned from "new study notifications" included in regularly scheduled network member newsletters or in a direct mailing to network members (please see Attachment A: Recruitment Materials). Each specific card will be included in the newsletter or in the direct mailing, accompanied by a Study Interest Sheet as well as the Study Instruction Sheet. Please see Appendices A: Recruitment Materials, and B: Data Collection Study Information Sheets.

On receipt of a Study Interest Sheet marked with "Yes, I want to participate" or "Maybe, please contact me" a CREC-certified research assistant will contact the clinician via the method selected on the study interest sheet to answer questions, confirm interest in participation, and confirm contact information. The research assistant will mail the clinician a packet including the clinician consent form, information sheet, a supply of cards, and a return envelope. The research assistant and study PI will be available to answer any questions.

\section{Data Collection}

The data collection includes each clinician filling out a data collection card with de-identified data immediately after eligible patient visits as part of their routine charting for each situation that falls under the purview of a specific card during a time period. Clinicians will be asked to record the number of patients seen by the clinician during the period of data collection, to provide a denominator for quantifying the frequency of the phenomena being studied.

The clinician will return the data collection cards to the research staff in a pre-paid envelope. Clinicians will instructed to not record any identifying information about patients on the data collection cards, via written and oral instructions. In the case that a data collection card is returned with identifying information, the information will be blacked out with a permanent marker, a photocopy of the data collection card (including the strikeout) will be made, and the original data collection card will be disposed of in a locked bin for secure shredding.

Following data analysis, clinicians desiring feedback will receive their own specific aggregate level data as well as a comparison of their data to all study data.

This study does not involve the collection of data from existing records or data often referred to as "on-the-shelf."

Final copies of all study materials (Direct Mailing Materials, Card Study Instruction Sheets, and data collection cards) for all specific card studies will be submitted to the IRB for approval before use.

\section{Study Parameters}

Percentage of clinicians who volunteer to gather data

Percentage of clinicians to successfully complete data collection

Percentage of complete data collection cards

Practice size

Clinician years in practice

Clinician type (MD, DO, NP, PA, or other)

Percentage of clinicians who request feedback reports

All specific items included in the data collection cards. Please see Attachment B: Card Instruction Sheets and Data Collection Cards for the remaining items tied to specific cards.

\section{Risks and Discomforts}

No intervention will be delivered as part of this research study. Confidentiality issues, especially 
those related to protected health information, could pose the greatest risk to patients in this study of usual care. The confidentiality of patients in this study is protected through steps such as collection of only de-identified patient data. The privacy of participating clinicians will be protected by the use of code identifiers on the data collection cards. Only the research staff will have access to the file linking identifiers with clinicians.

\section{Benefits to Subjects and Society}

There are no direct benefits to the patients from which data will be collected. These patients will be receiving usual care which will not be altered in any way by the study as the data collection form will be completed after the visit. The clinicians in the study may benefit from the receipt of aggregate data on patients in their practice, which will be provided at the completion of data collection. They may also benefit from the chance to be reflective with others about their practice. A greater understanding of the practice of primary care may result from the study, which would benefit society.

\section{Costs to Subjects}

There will be no costs for participating.

\section{Alternatives to Participation}

Alternatives to participation include participation in other primary care research studies or not participating in research studies.

\section{Payments to Subjects}

Study participants will not be compensated, though participating clinicians may consider the feedback on questions of clinical interest in a real-world setting to be valuable.

\section{Data Sharing and Confidentiality}

Collected data will have no direct or indirect linkages to patients' identities. According to the Privacy Rule, such nonidentifiable information can be released by medical practices for research purposes without patient authorization if approved by an Institutional Review Board.

Only CREC-certified staff will have access to completed surveys. Surveys will contain no patient identifiers but will contain a clinician ID number that will be assigned before mailing of the clinician packet. Each clinician's blank data collection cards will be pre-filled with the unique clinician ID. This
ID number is necessary to link the de-identified patient data with individual clinicians to provide feedback for those who desire feedback information. This feedback is a major component of participation for the clinicians. Surveys will be located in a secure building, in a locked file cabinet, located in a locked office. Electronic files are housed on a computer in a locked office connected to a secure network. The privacy of participating clinicians will be protected by the use of code identifiers on the data collection cards. Only the research staff will have access to the file linking identifiers with clinicians.

\section{Locations Where the Study Will be Conducted}

Data will be collected in the community practices which are part of the Practice-based Research Networks affiliated with Case Western Reserve University.

\section{Plan for Obtaining Informed Consent Practice Patients}

A waiver of patient informed consent is requested under 45 CFR 46.116(d). The research involves no more than minimal risk to the subjects. The waiver or alteration will not adversely affect the rights and welfare of the subjects. The research could not practicably be conducted without the waiver or alteration.

No identified information will be collected on patients. The proposed study cannot be practicably conducted without a waiver as the card study aims to characterize usual care as well as the ability of clinicians to capture de-identified data elements. The phenomenon typically emerges during the course of usual medical care, and stopping care to obtain consent would disrupt the phenomenon on which de-identified data are being collected. In addition, clinicians are often unable to obtain full informed consent as part of a usual care schedule without the assistance of an available research nurse. Research staff will not have access to patient contact information or patients and would not be able to obtain consent.

\section{Practice Physicians}

On receipt of a Study Interest Sheet marked with "Yes, I want to participate" or "Maybe, please contact me" a CREC-certified research assistant will contact the clinician via the method selected on the study interest sheet to answer questions, confirm interest in participation, and confirm contact infor- 
mation. The research assistant will mail the clinician a packet including the clinician mailed consent form, information sheet, a supply of cards, and a return envelope. The research assistant and study PI will be available to answer any questions. The clinicians will voluntarily complete and return the data collection cards.

\section{Request for Waiver of Signed Consent Under 45 CFR 46.117(c2)}

The research presents no more than minimal risk of harm to the subjects and involves no procedures for which written consent is normally required outside of the research context.

Clinician identity will be protected by the use of identifiers, which only research staff will have access to. Clinicians will be documenting information on usual care as part of their usual routine.

\section{Request for Waiver of HIPAA Authorization Requirement}

This is a request to use a limited data set as denoted under the Privacy Rule in the conduct of this research. Direct subject identifiers will not be collected and will not be present in the dataset; however, the limited data set will include the following elements: visit reason, treatment type, examination and diagnostic tests, and ambulatory visit dates. The limited data set will be only be used or disclosed by members of the research team.

The use of the limited data set involves no more than minimal risk to the privacy of individuals as research staff will not have access to patient identifiers. The information recorded will not extend beyond that which is needed for usual care.

The proposed study poses minimal risk to the privacy of the subjects because: (a) The limited dataset will be protected from improper use or disclosure in several ways. Research members will receive completed "cards" (please see Attachment B: Card Instruction Sheets and Data Collection Cards) containing de-identified data only. (b) No identifiers will be collected. The limited data set elements will be destroyed at the earliest opportunity consistent with the research, at the end of data collection. (c) The limited dataset elements will not be reused or disclosed to any other person or entity outside UHC other than those identified in the protocol, except as required by law, for authorized oversight of this research study, or as specifically approved for use in another study by an IRB.
The proposed study cannot be practicably conducted without a waiver of authorization as the card study aims to characterize usual care as well as the ability of clinicians to capture de-identified data elements. The phenomenon typically emerge during the course of usual medical care, and stopping care to obtain consent and HIPAA authorization would disrupt the phenomenon on which de-identified data are being collected. In addition, clinicians are often unable to obtain full informed consent and HIPAA Authorization as part of a usual care schedule without the assistance of an available research nurse. Research staff will not have access to patient contact information or patients and would not be able to obtain authorization.

\section{Data Analysis Plan}

Descriptive statistics will be used to examine the distribution of key outcome and predictor variables. Means and standard deviations will be computed for continuous variables and percentages for categorical variables. These data will be used to describe the study participants, address the descriptive research questions, and inform the statistical analysis. Clinicians will be provided with data specific to their own clinical practice as well as comparison to the entire study sample.

\section{Contact Information}

James J. Werner, PhD, Assistant Professor of Family Medicine, Department of Family Medicine, Research Division, 11001 Cedar Avenue, Suite 306, Cleveland, OH 44106; E-mail: james.werner@ case.edu; Michelle Hamilton, CCRP, Case Western Reserve University, Department of Family Medicine, Research Division, 11001 Cedar Avenue, Suite 306, Cleveland, $\mathrm{OH}$ 44106; E-mail: michelle.hamilton@case.edu.

\section{References}

1. Green, LA, et al. Results of reanalysis of the monthly prevalence of illness in the community and the roles of various sources of health care. N Engl J Med 2001;344:2021-202419.

2. Westfall JM, Mold J, Fagnan L. Practice-based research: "Blue Highways" on the NIH roadmap. JAMA 2007; 297:403-6.

3. Nutting PA, Beasley JW, Werner JJ. Practice-based research networks answer primary care questions. JAMA 1999;281:686-8.

4. Kutner JS, Main DS, Westfall JM, Pace W. The Practice-based Research Network as a model for 
end-of-life care research: challenges and opportunities: strategies for improving PBRN participation and quality of research. cancer control. 2005;12: 186-95.

5. Green LA. The weekly return as a practical instrument for data collection in office based research. Fam Med 1988;20:182-4.
6. Green LA, Hickner J. A short history of primary care practice-based research networks: from concept to essential research laboratories.

7. Green LA, Reed FM, Miller RS, Iverson DC. Verification of data reported by practices for a study of spontaneous abortion. Fam Med 1988;20: 189-91. 


\section{Newsletter Recruitment Language}

\section{New card Study Available}

Interested clinicians are invited to participate in a new card study. Please refer to the insert provided in this newsletter for more information.

As Always, your card study ideas are welcomed! Please use the request form via our website http://www.case.edu/med/pbrn/PEArequest.html or call the Clinical Research Facilitator (CRF) office at 216-368-4826.

\section{Direct Mailing Template}

\section{Dear CLINICIAN,}

The PBRN MEMBER NETWORK practice-based research network (PBRN) is participating in a new study looking at a SPECIFIC CARD INFORMATION.

In this study, clinicians will complete double-sided $3 \times 5$ index cards following visits with patients SPECIFIC CARD ELIGIBILITY for DATA COLLECTION TIME PERIOD. Following TIME PERIOD, the data collection cards and study instruction sheet may be returned to the research office in a provided envelope. A sample collection card as well as the study instruction sheet is included with this letter.

If you participate in the study, you will receive a confidential feedback report showing the aggregate responses of your patients on each survey item. Aggregate peer-comparison reports will also be provided, showing comparisons between your patients' responses and those of the entire study sample.

Please contact a Clinical Research Facilitator (CRF) with any questions at 216-368-4826 or by email at pbrn@case.edu.

Thank you for considering this request.

Sincerely,

James J.. Werner, Ph.D.

Assistant Professor of Family Medicine, School of Medicine

Case Comprehensive Cancer Center

Center for Reducing Health Disparities

Case Western Reserve University 
Card Study Interest Response Sheet

Clinician Name:

Practice Name:

Please Check the box that applies to you:

Yes, I want to participate

No, I do not want to participate

Maybe, please contact me so that I can get more information

Preferred Method of contact (please circle): email phone mail

Contact Information (phone, email, or address):

Is there an office manager or staff member who you would prefer we contact instead of contacting you directly?

Name:

Contact Information:

Please fax this form to (216)-368-4348

Please direct any questions to one of our Clinical Research Facilitators:

216-368-4826

pbrn@case.edu

Thank you for your time 
Instruction Sheet Template

\section{CARD STUDY TITLE: a NETWORK card study Instruction Sheet}

\section{Research Question \\ PLEASE SEE SECTION 2. - INDIVIDUAL CARD STUDY INSTRUCTION SHEETS. \\ Background \\ PLEASE SEE SECTION 2. - INDIVIDUAL CARD STUDY INSTRUCTION SHEETS.}

Card studies are one way for networks of clinicians to quickly gather information about a research question and promptly share what is learned.

\section{The Card}

Data collection will take place on the double-sided index-sized cards provided. These cards are designed to fit conveniently into any pocket. XX cards are provided for Time Period of data collection. Each card should take no more than one to two minutes to complete after the visit. At the end of each day, completed cards can be dropped into the study envelope provided by the research staff. Cards should be returned at the end of the data collection period in the provided postage-paid, addressed envelope.

\section{What you will need to do}

1. Choose a week when you will be seeing patients in your regular routine.

2. For that week, keep track of the total number of patients seen by you.

Please enter this number here

3. For every patient during the chosen week that INSERT SPECIFIC CRITERIA, fill out a card. Please do NOT write any identifying information on the card.

4. At the end of the week, use the provided envelope to mail this sheet and completed cards back to the research staff.

5. For questions, please call the principal investigator, James Werner, at 216-368-2996 or a Clinical Research Facilitator at 216-368-4826.

We have a few additional questions about you and your practice:

How many clinicians are in your practice?

How many years have you been in practice?

What type of clinician are you (MD, DO, NP, PA, etc)?

Would you like a copy of the study results (please circle)? Yes No

Would you like a comparison of your results with everyone else's (please circle)? Yes No

Thank you 


\section{Example Card Study Instruction Sheet and Data Collection Card}

\section{Identification of Development Delays: a Rainbow Research Network Card Study Instruction Sheet}

\section{Research Question}

How are developmental delays identified in infants and toddlers and what are the characteristics?

\section{Background}

The American Academy of Pediatrics (AAP) has recently issued a policy statement recommending that developmental surveillance be incorporated to every well-child health visit. Furthermore, they recommend that standardized developmental screening tests should be administered at these well-child visits. Practicing pediatricians have relied on their clinical intuition, patient exam, and observations thus far in detecting developmental delays. Examining how new developmental delays are identified will provide deeper insight into the utility of standardized instruments when compared to clinical intuition.

Card studies are one way for networks of clinicians to quickly gather information about a research question and promptly share what is learned.

\section{The Card}

Data collection will take place on the double-sided index-sized cards provided. These cards are designed to fit conveniently into any pocket. 10 cards are provided for 1 week of data collection. Each card should take no more than one to two minutes to complete after the visit. At the end of each day, completed cards can be dropped into the study envelope provided by the research staff. Cards should be returned at the end of the data collection period in the provided postage-paid, addressed envelope.

\section{What you will need to do}

1. Choose a week when you will be seeing patients in your regular routine.

2. For that week, keep track of the total number of patients seen by you.

\section{Please enter this number here}

3. For every patient during the chosen week who you screen for a developmental delay, fill out a card. Please do NOT write any identifying information on the card.

4. At the end of the week, use the provided envelope to mail this sheet and completed cards back to the research staff.

5. For questions, please call the principal investigator, James Werner, at 216-368-2996 or a Clinical Research Facilitator at 216-368-4826.

We have a few additional questions about you and your practice:

How many clinicians are in your practice?

How many years have you been in practice?

What type of clinician are you (MD, DO, NP, PA, etc)?

Would you like a copy of the study results (please circle)? Yes No

Would you like a comparison of your results with everyone else's (please circle)? Yes No

Thank you 
$\underline{\text { Identification of Developmental Delays Card Study Data Collection Card }}$

Front

Identification of Developmental Delays

Patient Age:

Patient Sex:

What initially caused you to be concerned that your patient might have a developmental delay?

$\square$ Clinical intuition $\quad \square$ Clinical exam $\square$ Clinical observation

$\square$ Parental concern $\square$ Developmental screen (which one?)

$\square$ Other

What developmental delay were you concerned about?

Back

Did you do anything about the developmental concern during this visit?

What follow-up is planned for this concern?

What was the level of parent/caregiver concern during the visit?

$\square$ Not at all concerned $\quad \square$ Somewhat concerned $\quad \square$ Very concerned

What was your level of concern during the visit?

Not at all concerned $\quad \square$ Somewhat concerned $\quad \square$ Very concerned

Additional Comments: 